\title{
Fenómenos naturales y amenazas latentes en la localidad de Volcán, Quebrada de Humahuaca, provincia de Jujuy, Argentina
}

\section{Natural phenomenon and latent threats in the town of Volcán, Quebrada de Humahuaca, Jujuy province, Argentine}

ARTÍCULO

\begin{abstract}
Álvaro Patricio Villarrubia Gómez
Universidad Nacional de Jujuy, Argentina. Contacto: alvaropvg@gmail.com
\end{abstract}

Recibido: agosto de 2021 Aceptado: octubre de 2021

\section{Resumen}

El presente artículo propone el estudio de caso de la localidad de Volcán, provincia de Jujuy, República Argentina en vinculación con conceptos relacionados como la vulnerabilidad, los fenómenos naturales y los riesgos. Para ello, se desarrollan cuestiones metodológicas orientadas a circunscribir la investigación, introducir en la temática, desarrollar antecedentes y un breve marco teórico. La problemática es presentada brindando una localización espacial del lugar donde se produce el fenómeno. Seguidamente, se considera la amenaza exponiendo características de la misma a través de una cronología, detallando consecuencias y los problemas que causa. Los conceptos de vulnerabilidad, riesgo y las capacidades relacionadas con su prevención son analizados en vinculación con el estudio del caso de Volcán. Sobre los conceptos trabajados se evidencia la necesidad de continuar realizando estudios e investigaciones sobre eventos adversos que poseen gran vinculación con la sociedad y sus vulnerabilidades, además de emerger la importancia de sensibilizar a las poblaciones sobre el conocimiento de acciones a realizar ante eventos de estas características.

Palabras clave: Vulnerabilidad, fenómenos naturales, amenaza, Volcán, Jujuy.

\section{Abstract}

This article proposes a case study of the town of Volcán, in the province of Jujuy, Argentina, in connection with related concepts such as vulnerability, natural phenomens and risks. For this, methodological questions are developed aimed at circumscribing the research, 
introducing the subject, developing antecedents and a brief theoretical framework. The problem is presented by providing a spatial location of the place where the phenomenon occurs. Next, the threat is considered, exposing its characteristics through a chronology, detailing the consequences and the problems it causes. The concepts of vulnerability, risk and the capacities related to their prevention are analyzed in connection with the study of the case of Volcán. Finally, reflections on the case addressed and on the concepts worked are presented, evidencing the need to continue carrying out studies and research on adverse events that are closely linked to society and its vulnerabilities, in addition to highlighting the importance of sensitizing populations about it knowledge of actions to be taken in the event of events of these characteristics.

Keywords: Vulnerability, natural phenomenon, threats, Volcán, Jujuy.

\section{A modo de introducción}

Desde sus orígenes, la humanidad se ha visto afectada por los desastres naturales, con pérdida de vidas y bienes materiales. Los fenómenos naturales, cuya realización da lugar a los desastres son de dos tipos: geológicos y meteorológicos. Los deslizamientos, desprendimientos, flujos y avalanchas consisten en el desplazamiento a favor de la pendiente y por efecto de la gravedad de masas de materiales que pueden moverse kilómetros desde su punto de origen (Alonso, 2011). Un ejemplo de estos fenómenos naturales que provocan desastres se encuentra en la localidad de Volcán, en la provincia de Jujuy.

La localidad de Volcán se nuclea alrededor de la estación ferroviaria homónima, perteneciente al riel "General Belgrano", sobre el margen occidental del Rio Grande, en la hermosa y pintoresca Quebrada de Humahuaca, hoy inscripta en la lista de Patrimonios de la Humanidad. Esta localidad, portal de ingreso a la Quebrada, dista a unos 30 kilómetros de la ciudad de San Salvador de Jujuy, por la Ruta Nacional $N^{\circ} 9$ o también denominada Panamericana (Villarrubia Gómez, 2012).

En el lugar es posible observar un amplio abanico formado por materiales aluvionales que han descendido por una quebrada que drena hacia el Rio Grande desde las escarpadas laderas occidentales, a más de 4000 metros sobre el nivel del mal (Alonso, 2011). Con la llegada de las lluvias de enero y febrero, estos materiales se embeben y fluyen aguas abajo como un barro espeso, cargado de trozos rocosos de todo tamaño. Es así que todos los habitantes de la provincia de Jujuy conocen los riesgos a los cuales está expuesta la población que vive en la localidad de Volcán y alrededores.

En este sentido, existieron en la historia, algunas remociones de suelo que provocaron eventos que afectaron a los pobladores de Volcán. Algunas de estos torrentes se produjeron en los años 1923, 1930 y 1945, provocando notorios daños a la vía férrea y al poblado 
(Alonso, 2011). Recientemente, el 10 de enero de 2017 se produjo un nuevo deslizamiento que provocó no solo daños materiales (como en otros años) sino que provocó muertes y desapariciones.

Es por esta situación que resulta pertinente realizar un análisis para conocer la historia de la problemática, además de indagar sobre el estado actual de la misma y los efectos o impactos sobre las poblaciones. Además, será necesario conocer también el papel o rol de la población local y de los entes gubernamentales, a fin de entender como la problemática afecta a la sociedad y cómo ésta posee capacidad para recuperarse y mejorar.

\section{La problemática latente}

El fenómeno peligroso que será objeto de estudio se encuentra representado por los aluviones o aludes de barro que se desarrollan en las proximidades de la localidad de Volcán, en el departamento Tumbaya, provincia de Jujuy.

Estos aludes son comunes en la zona de la Quebrada de Humahuaca, pero es el de Volcán el que presenta mayores exposiciones a la población, debido a la cercanía del espacio urbanizado. Es destacable, además, que el fenómeno a estudiar, no solo afecta a la población de Volcán, sino que también existen registros de años en que el alud ha llegado a afectar a poblaciones aledañas como Bárcena o Tumbaya, ambas distantes a 5 kilómetros al sur y norte respectivamente, de la localidad de Volcán.

El 10 de enero del año 2017, se produjo un alud de barro que dejó graves consecuencias en la localidad de Volcán: fallecieron cuatro personas y se reportaron más de mil evacuados, muchos de ellos perdiendo todas sus pertenencias.

Sin embargo, este evento no fue el primero en ocurrir en esta localidad y con similares características, sino que, en los años 1923, 1930 y 1940 Alonso (2011) ya explica que se producían estos eventos. Asimismo, existieron otros aluviones de similares características en el año 1945. El Ingeniero Quintana (2017) explica que:

El evento de mayor trascendencia relacionado con el tema, se produjo en la localidad de Volcán en marzo de 1945 donde el alud, de características extraordinarias que descendió por el denominado Arroyo del Medio, produjo un taponamiento sobre el Río Grande, que trajo como consecuencia el anegamiento de toda la localidad. (p. 8)

En este sentido, Quintana (2017) reflexiona que:

Desde ese hecho hasta la fecha la Quebrada de Humahuaca estuvo marcada, en casi todos los veranos (períodos de lluvias), por eventos de este tipo, a tal punto que la traza de la Ruta Nacional 9 fue modificada en gran parte de su recorrido, como ser los tramos: Río Reyes -Yala, Yala -Lozano, Lozano -León, León - Barcena (Corte Azul y Corte Colorado), Barcena - Volcán, Tumbaya - Maimará, Maimará - Tilcara (Quebrada de Huichaira) Huacalera - Uquía (Quebrada de Yacoraite) . (p. 8)

\section{Entre quebradas y amenazas constantes}


La Quebrada de Humahuaca se encuentra conformada por los departamentos de Humahuaca, Tilcara y Tumbaya, que en conjunto conforman un itinerario cultural y natural excepcional. Es oportuno explicar que la Quebrada se extiende a lo largo de varios kilómetros, acompañados por el Río Grande, perteneciente a la cuenca del Bermejo (Albeck y González, 1994) y que esta zona, constituye, además, un ambiente de fuertes contrastes: vegetación abundante en el sur, colindando con un fuerte cambio de paisaje marcado por la aridez y presencia de cardones en la zona central y norte de la quebrada.

Hacia los primeros años del nuevo milenio, desde el Estado Nacional y Provincial, comenzaron a efectuarse gestiones desde un proyecto que aparentemente ostentaba un modelo participativo de gestión, que derivaron en la incorporación de una región de la provincia: "La Quebrada de Humahuaca" a la lista de Patrimonio Mundial de la UNESCO, en el año 2003. Este reconocimiento, asociado vertiginosamente a programas de desarrollo turístico, generó una re-conceptualización del patrimonio como fuente de ingresos económicos. (Montenegro, 2011)

Puede observarse que los eventos producidos en los últimos años, tanto de orden natural como de origen humano, presentan un elemento en común: se producen situaciones que afectan al ambiente, al territorio y a la sociedad. Puede decirse, entonces, que estos riesgos afectan a las sociedades en mayor o menor medida.

Desde esta perspectiva, cada sociedad, históricamente, ha tomado un territorio para su desarrollo, como ámbito del cual extraer recursos y como lugar donde construir su propio hábitat. Los procesos centrales por los que un ambiente es utilizado por las sociedades actuales son los de apropiación (quién es el dueño, el propietario) y de valorización (cómo se transforman los recursos).

Se entiende que el territorio es uno de los espacios en los que el hombre ha influido de manera más determinante. Desde sus orígenes, la humanidad ha tomado de la Tierra todo lo que necesitaba; la acción humana ha conseguido a lo largo del tiempo, y sobre todo en el siglo XX, alterar física, química y biológicamente la Tierra, provocando distintos efectos, como son: agotamiento, erosión, desertización, deterioro y envenenamiento de la piel de la Tierra, a la que llamamos suelo, además de los impactos socio-culturales sobre las mismas poblaciones.

Sin embargo, existen algunos fenómenos naturales en la Quebrada de Humahuaca que, al sumarse con falta de infraestructura y obras, falta de estudio y conciencia sobre las consecuencias que provoca, generan situaciones que muchas veces culminan en catástrofes, tal como es el caso del alud de Volcán del año 2017.

Por su parte, los deslizamientos de tierra son causados por alteraciones en el equilibrio natural de una pendiente. Pueden ocurrir durante lluvias torrenciales o producirse luego de sequías, terremotos o erupciones volcánicas. Los aludes de barro se producen cuando el agua se acumula rápidamente en la tierra y causa un repentino aumento de rocas, tierra y 
detritos saturados con agua. Por lo general, los aludes de barro comienzan en pendientes empinadas y pueden ser desencadenados por desastres naturales.

En este sentido, Tarbuck y Lutgens (2005) explican que:

El exceso de pendiente es otra causa de muchos movimientos de masa. En la naturaleza hay muchas situaciones en las que hay exceso de pendiente. Una corriente fluvial que socava la pared de un valle y las olas que golpean contra la base de un acantilado no son sino dos ejemplos familiares (p. 428).

Asimismo, a estas situaciones se les adiciona otros factores que contribuyen a los movimientos y remociones de masa:

La gravedad es la fuerza que controla los procesos gravitacionales. Otros factores que influyen o desencadenan los movimientos pendiente abajo son la saturación en agua del material, el exceso de pendiente de las laderas, más allá del ángulo de reposo, la eliminación de la vegetación y el temblor de tierra producido por los terremotos (Tarbuck y Lutgens, 2005, p. 443).

Por otro lado, también puede decirse que además de las causas físicas, las áreas empinadas en las que los incendios forestales o cambios en el terreno por parte del hombre debido al desarrollo de diversas actividades son particularmente propensas a los deslizamientos de tierra durante y después de lluvias. De esta manera, puede entenderse que los aludes de barro o deslizamientos de tierra, que son fenómenos naturales, pueden convertirse en problemáticas al momento de afectar a las sociedades humanas.

Resulta importante entender conceptos relacionados a esta amenaza. Wilches Chaux (1993) define a la vulnerabilidad como "la incapacidad de una comunidad para absorber, mediante el autoajuste, los efectos de un determinado cambio en su medio ambiente, o sea su inflexibilidad o incapacidad para adaptarse a ese cambio". Además, Pereyra (2020) afirma que "cuando la comunidad no puede transformar su estructura, adecuar sus partes y redefinir la orientación de sus procesos en un contexto que lo exige, ya sea ambiental, económico o social, se origina el desastre". Esto sucedió hace unos años en el caso que se está estudiando, debido a que la comunidad estaba consciente que podían ocurrir desastres naturales, pero no realizó cambios importantes.

Teniendo en cuenta la propuesta de Wilches Chaux (1993) se aborda el primer enfoque, tomando al hombre como ser humano y como ser natural y entendiendo que sus acciones pueden detonar situaciones de riesgo y desastre ocasionando desastres naturales. Se toma este enfoque, debido a que la zona donde se encuentra emplazada la localidad de Volcán, posee una gran historia de asentamiento por parte de comunidades de hasta 10.000 años de antigüedad, por lo que su establecimiento actual no responde al uso de recursos que provoque esta situación de vulnerabilidad (tal como propone el segundo enfoque).

Desde los ángulos o perspectivas propuestas por Wilches Chaux (1993), se pueden abordar: 
- La Vulnerabilidad Natural y Física, estudiando las condiciones naturales y características ambientales propias del lugar. A ello, se sumarán las características físicas de la zona de riesgo (análisis de infraestructura, casas, materiales, etc.). Esto se realizó en los primeros apartados, y se está reforzando en los apartados siguientes.

Es importante resaltar que el poblado de Volcán se encuentra al pie de un gran cono de deyección, por eso su análisis se deberá realizar primeramente desde estos ángulos.

- $\quad$ La Vulnerabilidad Social y Cultural: será pasible de análisis las características del grupo social y sus capacidades para afrontar estos hechos.

Además, se pretende relacionar también con las cuestiones ideológicas y culturales, en el sentido que el asentamiento tiene una estrecha vinculación con la historia de los pueblos originarios de la región.

- La Vulnerabilidad Política: toma un papel importante, donde se deben analizar el rol de los organismos políticos y de gestión, y cómo éstos reaccionan ante las situaciones vulnerables.

Finalmente, resulta importante entender que los movimientos en masa pueden producirse donde ya hubo antecedentes históricos de los mismo (en Volcán, por ejemplo) o donde nunca los hubo antes (en este último no se podría predecir ni mucho menos prevenir).

Alonso (2011) por su parte, explica que donde ya ocurrieron, se sabe que pueden volver a repetirse, pero no se sabe cuándo. Sin embargo, pueden establecerse acciones para poder soportar el fenómeno y no provocar un desastre.

Lavell y Franco (1996) definen al desastre como producto y como proceso. Desde un primer enfoque el desastre es visto como algo emergente, sorpresivo, imprevisto y el énfasis de la acción se centra en la emergencia, en la visión como proceso se lo caracteriza como algo latente de manera permanente que, en algún momento, por la conjunción de distintos factores detona. Estos autores se refieren al continuum de desastre, al hacer mención a una sociedad que convive con riesgos latentes que en algún momento detonan, pero que por sus condiciones sociales y económicas conviven con la posibilidad de un desastre de manera permanente.

\section{Presentación formal de Volcán}

La localidad de Volcán se fundó en el año 1905 con el trazado de la línea ferroviaria que ascendió a la Quebrada. La estación, que fue la última en el recorrido ferroviario de la región, se complementó con un importante taller de reparaciones. 


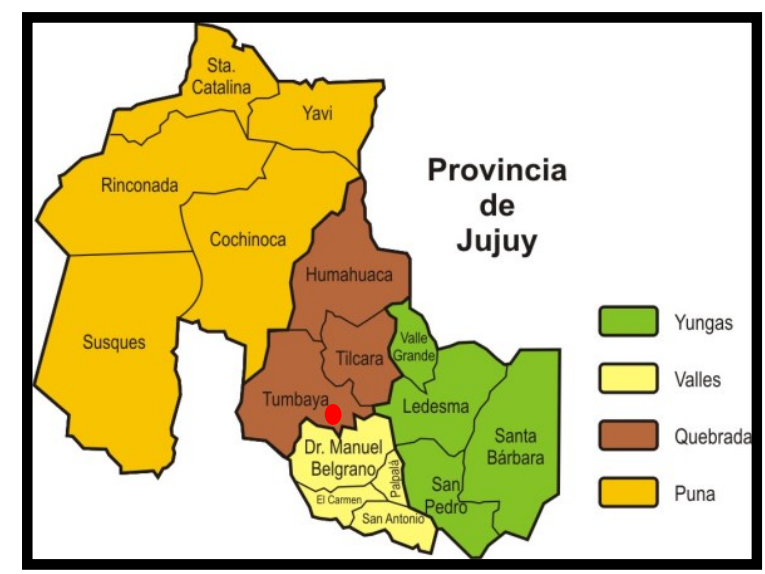

Figura N 1: Mapa de Localización de Quebrada y Volcán. Fuente: Elaboración propia.

De acuerdo con los datos del Censo de 2010 del INDEC, la localidad cuenta con 1731 habitantes. El clima es semi-árido y en invierno desciende mucho la temperatura. Soplan vientos de julio a agosto, con pocas lluvias de diciembre a abril. Es una zona de transición del clima templado de los valles y el árido de la quebrada.

El suelo en la zona, es árido. Sin embargo, se cultivan en pequeña escala algunos cereales como alfalfa, maíz, trigo, cebada, y también papa y vid; frutales tales como duraznos, manzanas y peras. Las especies forestales presentes son el sauce, toya y algarrobo. Entre las actividades agropecuarias que se evidencian, se observa cría de ganado caprino y bovinos, todos para el mercado interno o local.

Desde comienzos de verano se producen en la zona fuertes precipitaciones que ocasionan el desprendimiento de tierra y piedras que se deslizan en forma de aludes sobre la ladera de los cerros inmediatos a la localidad. En ciertas ocasiones, estos aluviones han ocasionado cortes de la Ruta Nacional 9 y daños en las viviendas e instalaciones, con importantes pérdidas económicas y riesgo para la población. Desde comienzos de la década de 1970 está instalada en la localidad una planta de producción de cal y derivados, principal abastecedora de estos productos para la región del noroeste argentino y del sur de Bolivia. La calera "Los Tilianes" se asienta en la ladera del cono de deyección del arroyo del Medio, donde descienden los aludes de barro.

Entre los cambios que pueden observarse se evidencia que, en parte de las instalaciones en desuso del Ferrocarril General Belgrano, se emplazó la Feria Campesina de Artesanos de la Quebrada y Puna, un espacio donde artesanos de varias localidades de la zona ofrecen sus productos elaborados en arcilla, madera de cardón, cueros, piedra, lana de oveja y de llama, reproducciones de cerámicas y de grabados y pinturas de yacimientos arqueológicos de la región

Dentro de los atractivos turísticos de la localidad de Volcán, pueden mencionarse: 
- La Laguna de Gronda que se encuentra a cinco kilómetros de Volcán, ubicada especificamente en el paraje Chilcayoc. Se trata de una laguna privada a la cual se puede tener acceso, previa autorización

- El Pucará de Volcán está ubicado al norte de la localidad sobre una altitud aproximada de 2700 m.s.n.m, reflejando lo que en algún momento supo ser un poblado indígena organizado, y constituye el primer pucará de la zona de la Quebrada de Humahuaca. (Cremonte y Scaro, 2010)

\section{La amenaza de Volcán}

El proceso erosivo en toda la extensión de la Quebrada, es un factor natural que evidentemente se produjo a lo largo de la historia geológica de la región, donde la intensidad de las lluvias juegan un papel fundamental, ya que constituye el ingrediente desencadenante en la producción del fenómeno, canalizando sus efectos por las redes de desagües naturales conformadas por las quebradas que buscan descargar el material acumulado en la zona alta de las mismas hacia las regiones más bajas, utilizando como medio de trasporte el agua procedente de las precipitaciones.

Como se mencionó anteriormente, la localidad de Volcán está ubicada en el departamento de Tumbaya y se localiza en el inicio de la Quebrada de Humahuaca, provincia de Jujuy. Alonso (2011) explica que "en el lugar, es posible observar un amplio abanico formado por materiales aluvionales que han descendido por una quebrada que drena hacia el Rio Grande desde las escarpadas laderas occidentales, a más de 4.000 msnm" (p. 167).

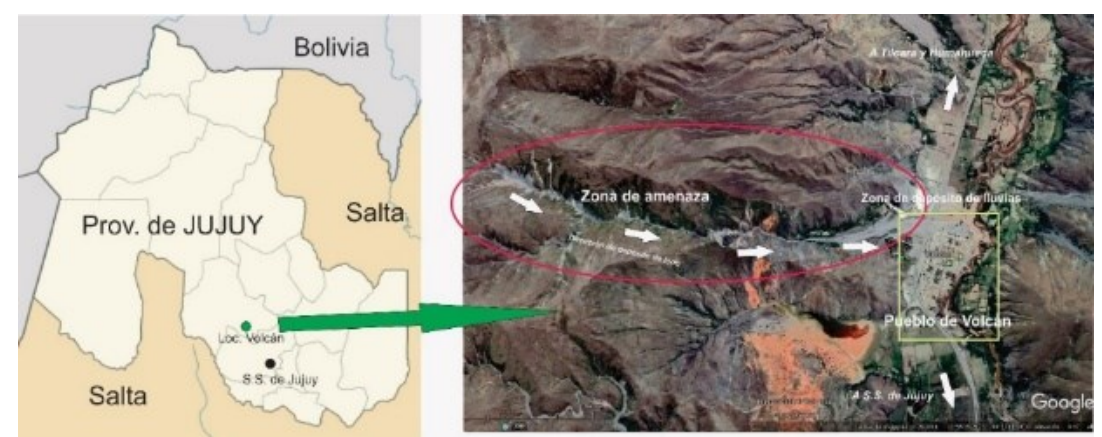

Figura N²: "La provincia de Jujuy y la amenaza de Volcán"- Fuente: Elaboración propia.

Como puede observarse, en la proximidad de la localidad de Volcán, existe un enorme cono de deyección formado precisamente por los aluviones que se suceden todos los años, cortando la ruta por horas (y antes, la circulación de los trenes). Pesadas maquinas viales deben entonces aplanar el camino pasando sobre el material recién depositado.

Los "volcanes de barro", son el nombre que se les propone regionalmente a las coladas de fango. Alonso (2011) explica que se producen por la especial situación en las laderas andinas, donde concurren situaciones de fuertes pendientes, rocas de distintas naturalezas litológicas, falta de cubierta vegetal, exposición a los agentes meteóricos, una larga estación seca, lluvias concentradas en el verano, etc. Con la llegada de las lluvias de enero y febrero, 
los materiales sueltos se embeben y fluyen aguas abajo como un barro espeso, cargado de trozos rocosos de todo tamaño. Si a estas condiciones, le sumamos que el pueblo de Volcán se asienta en la base del cono de deyección donde se depositan las coladas de fango, estamos frente a una amenaza latente

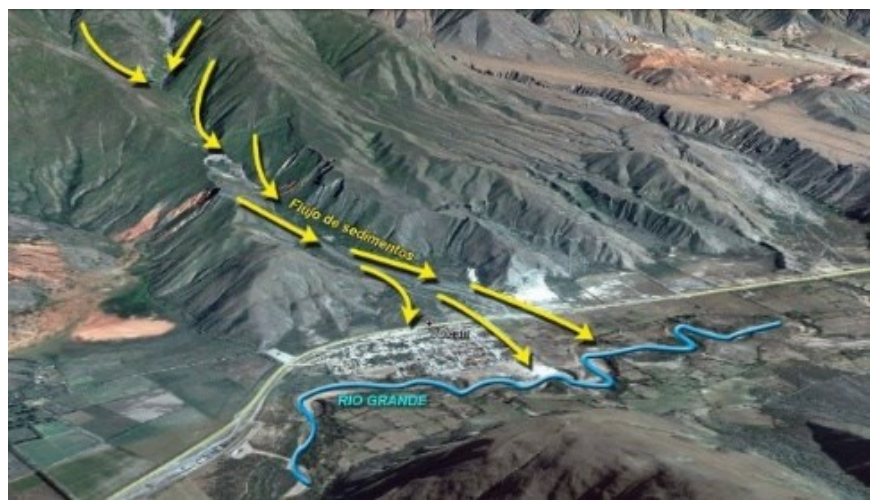

Figura N³: "El alud de Volcán" - Fuente: Diario digital jujuyalmomento.com

En 1943, el doctor Horacio Harrington describió que en ese año hubo una remoción de unos tres kilómetros cúbicos de material que se desplazó quebrada abajo, alcanzando velocidades entre 10 y 15 kilómetros por hora (Alonso, 2011). El torrente de "Volcán" es por sus características, el más impactante de los que ocurren en el norte argentino y un ejemplo de clase mundial citado en manuales internacionales, según explica Alonso. Asimismo, se puede decir que el último registro que se tiene de la activación de la amenaza del volcán, fue el día 10 de enero de 2017. Ese día, luego de una imprevista lluvia, el alud de lodo y fango arrasó el pueblo de Volcán, provocando la muerte de cuatro personas y dejando a más de mil evacuados.

\section{Identificación, caracterización y tipo de amenaza:}

En ciertos lugares de la montaña, las fuertes lluvias pueden provocar aluviones de barro: tierra, pedregullo y agua mezclados que forman una masa viscosa color marrón, que por su similitud con la lava volcánica es llamada "volcán" (Kirbus, 2006). Este fenómeno, se transforma en amenaza, debido a que los aluviones de barro, ocasionalmente, ingresan al pueblo de Volcán, provocando pérdidas económicas, daños materiales e incluso, en algunos años, muertes.

De acuerdo con lo propuesto por la Estrategia Internacional para la Reducción de Desastres (2004) la amenaza estudiada, corresponde al tipo:

- Amenaza Natural: Procesos o fenómenos naturales que tienen lugar en la biosfera que pueden resultar en un evento perjudicial y causar la muerte o lesiones, daños materiales, interrupción de la actividad social y económica o degradación ambiental. Dentro de esto, el caso de estudio se sub-clasifica también como: 
- Amenaza Hidrometeorológica: Procesos o fenómenos naturales de origen atmosférico, hidrológico u oceanográfico, que pueden causar la muerte o lesiones, daños materiales, interrupción de la actividad social y económica o degradación ambiental. Aquí se encontraría, como ejemplo el flujo de lodo.

- Amenaza Geológica: Procesos o fenómenos naturales terrestres, que puedan causar pérdida de vida o daños materiales, interrupción de la actividad social y económica o degradación ambiental. Dado el caso de estudio, también se ubicaría aquí debido a que se presentan movimientos de masas, deslizamientos, desprendimiento de rocas, etc.

Por su parte, Pereyra (2009) adiciona que también pueden entenderse a las amenazas en vinculación con las acciones de las sociedades, planteando así la existencia de amenazas socionaturales las cuales también se relacionan con el caso de Volcán, en tanto:

Vinculan las prácticas humanas con los rasgos ambientales y se caracterizan por procesos de degradación ambiental, carencia de infraestructuras, inadaptabilidad al entorno. Son el reflejo de los problemas ambientales y parten de la reconversión de recursos en amenazas a partir de las transformaciones territoriales. (p. 8)

\section{Expresiones territoriales del riesgo por deslizamientos}

Toda la región de la Quebrada de Humahuaca presenta riesgo por las amenazas de los aluviones o coladas de fango. Sin embargo, en el espacio específico de Volcán, y teniendo en cuenta los registros de daños en años anteriores, los aluviones de barro perjudican no solo al poblado de Volcán, sino que sus consecuencias también se evidencian en zonas aledañas como Tumbaya, al norte, o Bárcena al sur.

De esta manera puede discriminarse a las poblaciones afectadas de la siguiente manera, teniendo en cuenta el Manual para la Evaluación de Desastre de la CEPAL (2014):

- Población afectada primaria: Bajo esta clasificación se incluye a las personas que sufren los efectos directos del desastre: víctimas mortales, heridos e incapacitados (víctimas primarias traumáticas), albergados, desplazados y quienes sufren daños de su propiedad como consecuencia directa e inmediata del desastre. Este segmento de población es el que se encuentra en el territorio afectado en el momento del siniestro. En este caso, la población afectada primaria corresponde a la del poblado de Volcán.

- Población afectada secundaria: Son aquellas personas que estaban dentro de los límites del territorio impactado y que sufren otras consecuencias del desastre, como, por ejemplo, pérdida de su empleo a causa de la destrucción de la empresa en la que trabajaban, o pérdida de ingresos en el caso de los comerciantes del territorio en cuestión que se ocupaban en actividades de mercadeo relacionadas con cosechas agrícolas destruidas. Entre estas poblaciones encontramos la de las localidades aledañas como Bárcena, Coiruro, Tumbaya e incluso otras más alejadas como León, Purmamarca, etc. 
La escala de probabilidad de ocurrencia del fenómeno es mediana - alta. Debido a la complejidad de los sistemas físicos en los cuales un gran número de variables puede condicionar el proceso, aun no se cuenta con técnicas que permitan modelar con alta precisión la probabilidad de ocurrencia del fenómeno.

Sin embargo, teniendo en cuenta variables como:

- Historia cronológica de los eventos producidos.

- Características ambientales y del clima de la región.

- Condiciones del suelo.

Puede entenderse que este fenómeno seguirá sucediendo, debido a que la región presenta características acordes para que este tipo de deslizamientos se produzcan en las épocas estivales.

La evaluación de la amenaza, en la mayoría de los casos, se realiza combinando el análisis probabilístico con el análisis del comportamiento físico de la fuente generadora, utilizando información de eventos que han ocurrido en el pasado y modelando con algún grado de aproximación los sistemas físicos involucrados. En otras palabras, para poder cuantificar la probabilidad de que se presente un evento de una u otra intensidad durante un período de exposición, es necesario contar con información, lo más completa posible, acerca del número de eventos que han ocurrido en el pasado y acerca de la intensidad que tuvieron los mismo.

Como se pudo evidenciar, existieron años donde los eventos aluvionales devastadores se desarrollaron casi ininterrumpidamente. En otras ocasiones, se evidenciaron cada dos o tres años. Finalmente, otros se produjeron cada varias décadas, por lo que resulta improbable predecir cuándo se originará un nuevo evento catastrófico. Lo que sí se puede estimar, es que todos los años existen lluvias en la región quebradeña, y estas precipitaciones provocan deslizamientos que, en situaciones de máximas lluvias, provocan los deslizamientos.

Puede decirse también que, algunos de los factores que influyen en la amenaza descripta son:

- Impacto minero - industrial: En las adyacencias de la localidad de Volcán, más específicamente en la zona del cono de deyección del alud, se encuentra asentada la calera "Los Tilianes". Esta empresa privada de capitales nacionales, está dedicada a la explotación de caliza para abastecer a toda la región del NOA. Está asentada en la localidad de Volcán desde el año 1960. Producen un efecto que influye en la amenaza debido a que extraen minerales de la zona y contribuyen, muchas veces, a la degradación de los suelos.

- Impacto atmosférico- efecto invernadero- calentamiento global- cambio climático: Estas situaciones también afectan a la amenaza, en el sentido que en la región, se 
observan años con grandes cantidades de precipitaciones. Esto, según los lugareños, es producto de los cambios climáticos que se observan en la región.

- Uso inadecuado de los recursos naturales: El uso irracional y desmedido de los recursos naturales, como, por ejemplo, los minerales o árboles, contribuye a que estos aluviones de barro se extiendan cada vez más y provoquen mayores daños a las poblaciones.

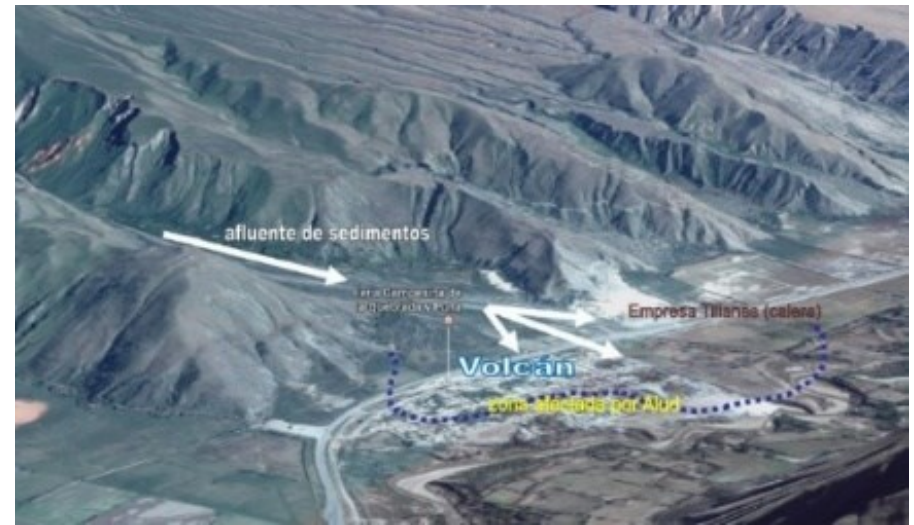

Figura $N^{\circ}$ 4: "Imagen satelital de la zona afectada por aludes y de la ubicación de la empresa Los Tilianes" - Fuente: Kirbus, F. (2017)

\section{Diferentes amenazas y peligros latentes}

Alonso (2011) explica que la capacidad de transporte de los ríos del noroeste argentino son más activos que en el pasado. El autor comenta que en el último medio siglo, hubo un enlentecimiento de los fenómenos de transporte y una aceleración de la acumulación o agradacion de los materiales en los lechos de los ríos.

El autor explica que:

Es sintomático escuchar a las personas mayores de Salta y Jujuy comentar que antes, y tomando como referencia cualquier puente, el río venía "allá abajo" y ahora el rio viene al borde del puente si es que no lo ha superado. Esta situación puede verse claramente en la Quebrada de Humahuaca, donde la mayoría de los puentes tuvieron que ser reemplazados por el aluvionamiento de los cauces (Alonso, 2011, p. 208).

Puede entenderse así que, además de los aluviones de barro, las crecidas o crecientes producidas por las grandes cantidades de precipitaciones caídas en la época estival, suman una nueva amenaza a la región de la Quebrada. Estas crecidas, provocan muchas veces que algunos poblados se inunden o queden aislados de otras poblaciones. 


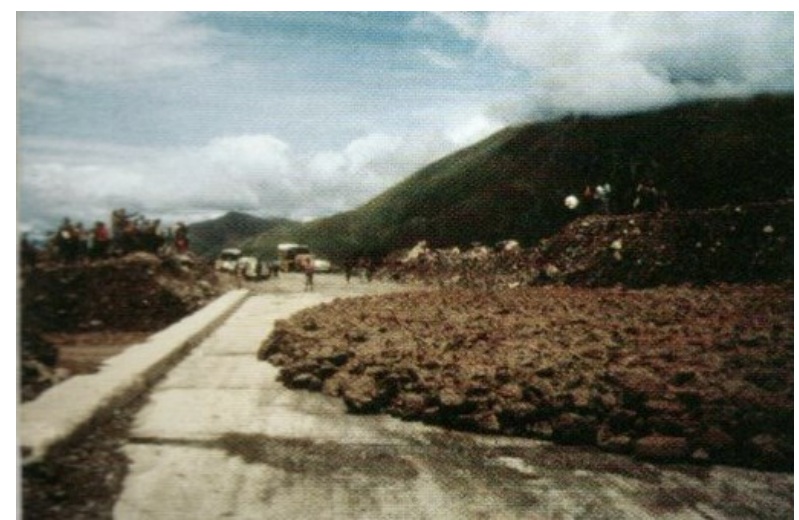

Figura N 5: "Corte sobre Ruta Nacional N 9 - Año 2005" - Fuente: Kirbus, F. (2006)

\section{La vulnerabilidad y la exposición}

La vulnerabilidad, como ya se explicó, hace referencia, según Romero y Maskrey (1993) a la capacidad que tiene un grupo de sufrir un daño y tener dificultad de salir de éste. En el caso de la exposición, está asociada a la capacidad de afrontamiento a un evento, a los riesgos naturales o al cambio climático (Coy, M. citado en Pereyra, 2009).

En el caso del poblado de Volcán, y la amenaza del alud, se pueden analizar indicadores socio-económicos (viviendas de los habitantes, falta de defensas, etc.); demográficos y de infraestructura (falta de defensas en las calles, canalizaciones, etc.) y de prevención y percepción del peligro (sensibilización de la población sobre los riesgos que tienen viviendo en el lugar, etc.)

Entendiendo que el riesgo es la probabilidad de que un fenómeno ocurra y provoque daños, se puede decir que la zona de Volcán se encuentra sobre un "cono de deyección" o "abanico aluvial", esto es una zona en donde los sedimentos arrastrados desde la montaña se depositan luego de las precipitaciones producidas en la alta montaña.

Si a esto, se suma que el clima predominante es el semi-árido, con precipitaciones que predominan únicamente en los meses de verano, además de ser abundantes y potentes, permiten que, en pocos minutos, se provoquen situaciones inesperadas como estos aludes.

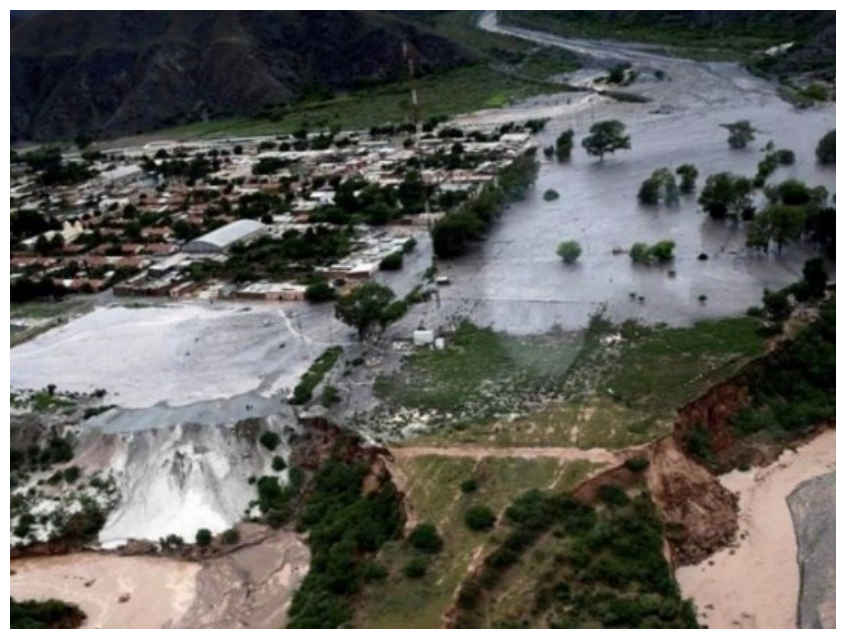


Debe entenderse, entonces, que ante la amenaza y riesgo constante que este espacio geográfico brinda, la población está tomando conciencia y sensibilizándose de los problemas que pueden suceder a futuro, con nuevos deslizamientos.

El pueblo de Volcán, en este sentido, es consciente de los riesgos a los que se enfrenta, pero también es un pueblo resiliente que puede reconstruirse, tal como lo documenta la historia.

Desde los ángulos o perspectivas propuestas por Wilches Chaux (1993), se pueden ampliar:

\section{La Vulnerabilidad Natural y Física:}

Permite entender que, los pobladores de Volcán están condicionados por la zona donde está emplazado el lugar. Esto es, un lugar con constantes deslizamientos, acrecentados en la zona veraniega. Es importante recordar que el poblado de Volcán se encuentra al pie de un gran cono de deyección.

Consecuentemente, se evidencian algunos avances para poder atender a estas cuestiones físicas y naturales, y están orientadas al desarrollo de infraestructuras de viviendas en zonas más alejadas al cono de deyección, y con materiales más resistentes.

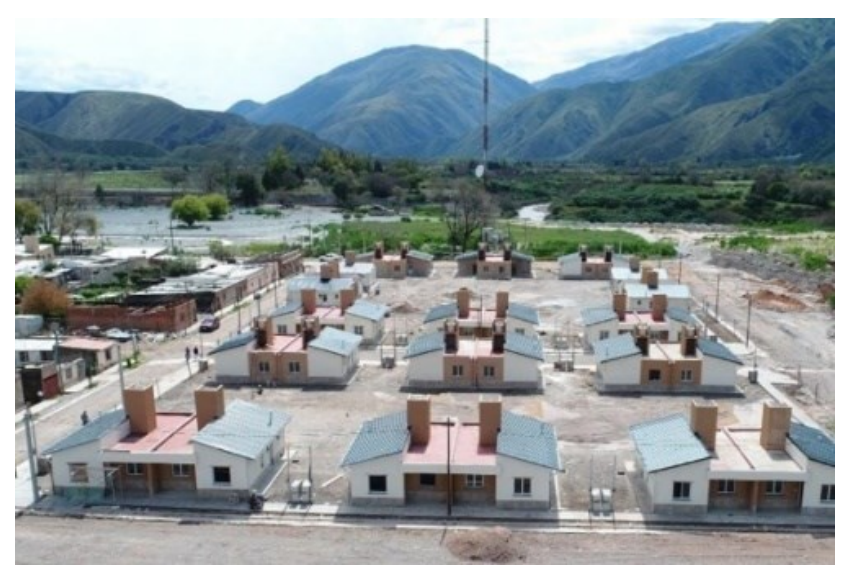

Figura N 7: "Nuevas viviendas en Volcán" - Fuente: Instituto de Vivienda y Urbanismo de Jujuy (2019)

Puede decirse que las viviendas reconstruidas, fueron emplazadas en una zona alejada al cono de deyección donde se producen las principales consecuencias. De esta manera, se está intentando dejar de exponer a los habitantes.

\section{La Vulnerabilidad Social y Cultural:}

Deben entenderse aquí, las características del grupo social y sus capacidades para afrontar estos hechos. Además, se debe relacionar también con las cuestiones ideológicas y culturales, en el sentido que el asentamiento tiene una estrecha vinculación con la historia 
de los pueblos originarios de la región. Los grupos asentados en esta localidad y en toda la región de la quebrada, poseen una valiosa ideología respecto a la tierra y sus poderes. La cosmovisión andina se encuentra fuertemente arraigada y los pobladores buscan asentarse en los territorios donde vivieron sus antepasados: abuelos, bisabuelos, etc.

Es por este hecho que, los pobladores no buscan transportarse a otras localidades para vivir y si deciden estar en sus territorios, aun conscientes de los riesgos a los que se exponen. Solo las poblaciones más jóvenes se dirigen a los grandes centros urbanos, buscando, de esa manera, nuevas oportunidades de vida.

\section{La Vulnerabilidad Política:}

Toma un papel importante, donde se deben analizar el rol de los organismos políticos y de gestión, y cómo éstos reaccionan ante las situaciones vulnerables.

El rol del gobierno provincial resulta importante, en tanto son los encargados de realizar obras de infraestructura para los jujeños. Muchas veces, el reclamo de los pobladores manifestaba la falta de estas obras en lugares tan críticos como lo es Volcán o las diferentes localidades de la quebrada.

Además, el gobierno local, provincial y nacional, tienen una deuda que se evidencia desde los grandes aluviones del siglo XX. Tal como se presenta en la publicación del diario El Día, del año 1945, se propone que el gobierno debe encarar la reconstrucción del pueblo de Volcán. Este pedido se volvió a escuchar en el año 2017, cuando los pobladores locales exigían al gobierno, la reconstrucción y soluciones definitivas ante la problemática de los aluviones de barro.
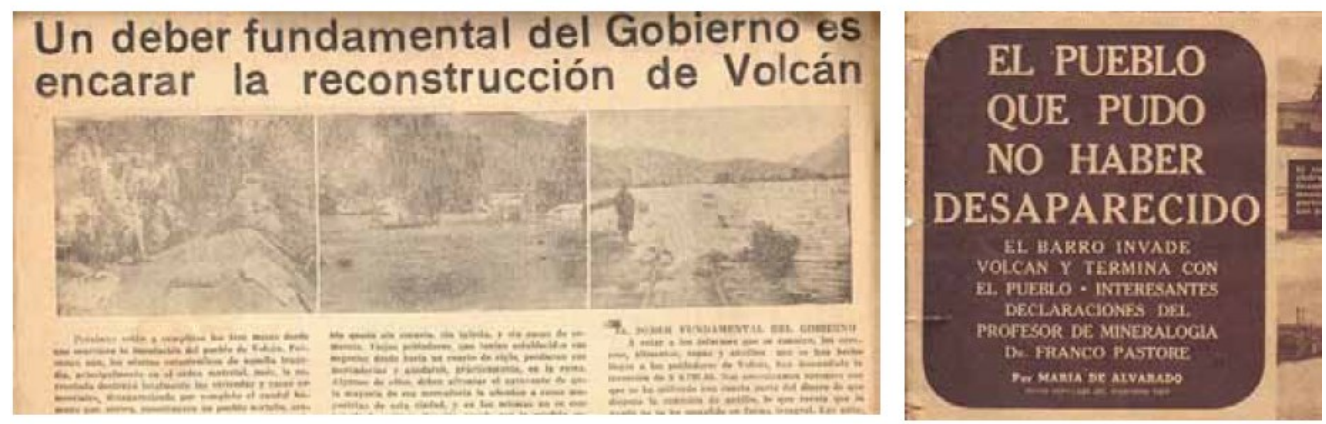

Figura N 8: "Vulnerabilidad de Volcán"-Fuente: Diario El Día (1945)

\section{Marco normativo sobre riesgos y desastres}

Durante el trabajo realizado, no se pudieron encontrar ni acceder a Ordenanzas Municipales que respondan a la temática de la gestión del riesgo. Se realizó, sin embargo, una búsqueda en los Boletines Oficiales de la Provincia de Jujuy, ampliando así la búsqueda a otras reglamentaciones o decretos provinciales que pudieran relacionarse con el tema, sin resultado positivo. 
El único decreto provincial que se dispuso para afrontar la situación del desastre en el mes de enero de 2017, fue el Decreto 3021-G-2017 que eximió del pago de servicios sanitarios y energía eléctrica a usuarios de Volcán, Bárcena y Tumbaya durante los meses de enero y febrero del mismo año por la situación del alud.

Aun así, resulta importante profundizar y continuar con la búsqueda de reglamentaciones y ordenanzas que pudieran haberse decretado en los últimos tiempos, y que propongan la gestión del riesgo y la realidad de Volcán como situaciones a tener en cuenta.

Como se mencionó anteriormente, si se encontraron planes desarrollados desde el gobierno provincial para afrontar los desastres provocados por el alud de barro. Estos planes buscaron la construcción y reconstrucción de viviendas, adoquinado de calles, nueva iluminación, entre otros. Sin embargo, no se encontraron propuestas y reglamentaciones que propongan soluciones o posibles acciones para afrontar los riesgos, entendiendo que la amenaza de un nuevo alud de barro es constante en la época estival.

En este sentido, todavía puede evidenciarse una mirada "fisicalista" en tanto se entiende a los desastres como "eventos físicos "naturales", tecnológicos o antrópicos con los cuales están asociados" ( p. 15) promoviendo una concepción que pone el peso de la responsabilidad en los eventos físicos en sí, jugando la sociedad un papel secundario. Así también, desde las diversas acciones u operaciones, se observa que estos eventos se presentan como "inmanejables", "impredecibles" o "inevitables" promoviendo nuevamente el enfoque "fisicalista" de los eventos.

\section{La gestión del riesgo}

Resulta oportuno proponer la gestión del riesgo como un proceso social cuyo objetivo no se limita a la preparación para una respuesta adecuada sino en la concepción y trabajo del desastre como proceso buscando controlar los factores de riesgo y asociado a procesos que tienden al desarrollo humano, económico, ambiental y territorial sostenible.

Un modelo que puede tomarse está relacionado con el modelo PAR (Modelo de Presión y Liberación de desastres). Blaikie (1996) se refiere a esta propuesta, explicando la importancia de integrar distintos factores que contribuyen a la construcción social del riesgo y buscan explicar el desastre desde la escala geográfica local, regional e internacional. Para el modelo PAR el desastre tiene lugar cuando dos fuerzas opuestas se intersectan, en este caso, la vulnerabilidad y la exposición a la amenaza. (Pereyra, 2020). Se presenta, a continuación, la aplicación del modelo PAR. 
MODELO DE PRESIÓN Y LIBERACIÓN (PAR)

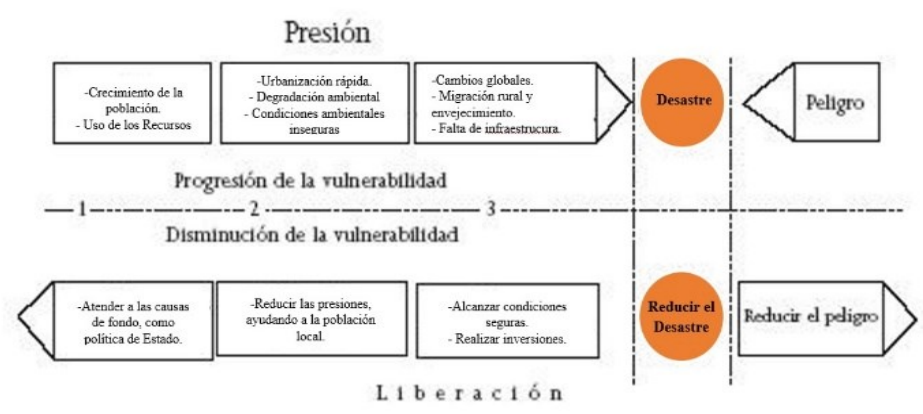

Figura N 9: Modelo PAR - Fuente: Elaboración propia en base al Modelo de Blaikie (1996)

El gráfico anterior puede explicarse entendiendo las:

- Causas de fondo: Están representadas por el primer recuadro, donde se propone que el crecimiento de la población y el uso de los recursos son las causas de fondo. Esto se relaciona con la idea del asentamiento de la comunidad en el territorio elegido. Históricamente, esta comunidad se asentó en el lugar debido al crecimiento que obligó a buscar un lugar específico que pudiera ofrecer el acceso a diferentes recursos. De esta manera, se elige el territorio de Volcán, debido a que se encuentra en un pasaje entre la quebrada y los valles, siendo así una importante vía de comunicación entre ambas regiones jujeñas.

Será importante analizar también como causas de fondo, las ideologías en tanto sistemas culturales de representación de los pueblos de Volcán. Por otro lado, también se podrán analizar los sistemas políticos y económicos como causas que provocan desastres.

- Presiones dinámicas: Están representadas por el segundo recuadro, que propone una urbanización acelerada, la degradación ambiental y las condiciones ambientales inseguras. Estas presiones, con el correr del tiempo, fueron acrecentándose y acelerándose, provocando diversos desastres en la historia del pueblo.

Además, pueden observarse como otras presiones, la falta de instituciones locales que puedan ayudar o asistir en cuestiones de desastres, el entrenamiento o el desarrollo de habilidades para poder enfrentar una situación de riesgo, entre otras.

- Condiciones inseguras: En el tercer recuadro se presentan sintéticamente las condiciones inseguras referidas al tema de trabajo. Entre éstas se destacan los cambios globales que existen en la sociedad de Volcán. Además, otra condición es la migración que realizan las poblaciones rurales hacia este poblado, acrecentando así la urbanización. Asimismo, se observa también un envejecimiento de la población, lo que sería una condición a tener en cuenta en el caso de posibles desastres. Puede analizarse también, la fragilidad del ambiente físico, así como las localizaciones peligrosas de viviendas o instituciones públicas (escuelas, terminales, hospital, etc.) 
Finalmente, la falta de infraestructura urbana adecuada se presenta como una de las condiciones más relevantes para entender la vulnerabilidad desde esta perspectiva.

Lavell y Franco (1996) explican que la oportunidad provocada por una serie de grandes desastres sucedidos y la clara necesidad de dar prioridad a la gestión de la respuesta inmediata tiene un corolario directo en los sectores o actores sociales que impulsan, guían o controlan la institucionalización temprana de las estructuras para la gestión de los desastres. Ante esta situación, pueden observarse algunos conflictos entre estos sectores:

- El sector gubernamental, a través de los ministerios provinciales (Infraestructura, vivienda, ambiente, etc.) propusieron reformas y reconstrucciones para los pobladores de la localidad de Volcán. Sin embargo, este sector gubernamental fue acusado de no realizar las obras preventivas, y que, a pesar de realizar planes de refacción del pueblo, no se observan, aún hoy, obras de defensa relevantes para afrontar un potencial alud.

- Población Local: Está representada por los diferentes pueblos de Volcán y alrededores. La población marcó su descontento al planteamiento del gobierno de trasladar parte de las viviendas a otros sectores de la localidad. Además, durante los años 2018 y 2019, diversos sectores del pueblo reclamaron la falta de obras de refacción propuestas por el gobierno provincial en el año 2017. Finalmente, las viviendas fueron inauguradas en el año 2019, dos años después del alud.

- Empresa minera: La empresa minera "Los Tilianes" se encuentra asentada sobre el cono de deyección del Volcán de lodo. Esto hizo que cuando se desarrolló el alud, la empresa y toda su infraestructura fue afectada en gran magnitud. Posteriormente, se buscó trasladar a la empresa a otro sector, generando conflictos entre el gobierno y la empresa privada, y no realizando ninguna acción al respecto.

\section{Institucionalidad de la gestión}

El ingeniero Quintana (2017) expone que:

A la luz de los acontecimientos, de esta naturaleza, producidos en los últimos años, que son de público conocimiento por la afectación de carácter grave para las poblaciones y las infraestructuras castigadas por el fenómeno, en mi opinión, resulta imprescindible la conformación de un grupo de profesionales multidisciplinario y debidamente calificado para el estudio, análisis, acciones sobre las cuencas, priorización de las mismas y consensuado de proyectos de distintas entidades que intervienen sobre las cuencas. (2017: p. 8)

Entendiendo este postulado, puede observarse que los eventos producidos en los últimos años, tanto de orden natural como de origen humano, presentan un elemento en común: se producen problemas que afectan al ambiente, acelerados por las acciones antrópicas. Además, necesitan profesionales que puedan atender y estudiar tales 
problemáticas, a fin de poder predecirlas, o mínimamente, estar preparados para soportarlas.

En este sentido, puede decirse que los riesgos afectan a las sociedades en mayor o menor medida. La propuesta del trabajo se enmarca en los postulados de Wilches Chaux (1993) quien define a la vulnerabilidad como "la incapacidad de una comunidad para absorber, mediante el autoajuste, los efectos de un determinado cambio en su medio ambiente, o sea su inflexibilidad o incapacidad para adaptarse a ese cambio". Por su parte, también resultarán relevantes los postulados de Pereyra (2020) quien afirma que "cuando la comunidad no puede transformar su estructura, adecuar sus partes y redefinir la orientación de sus procesos en un contexto que lo exige, ya sea ambiental, económico o social, se origina el desastre".

Es importante proponer la gestión del riesgo como un proceso social cuyo objetivo no se limita a la preparación para una respuesta adecuada sino en la concepción y trabajo del desastre como proceso buscando controlar los factores de riesgo y asociado a procesos que tienden al desarrollo humano, económico, ambiental y territorial sostenible. En este sentido, se analizará si se están realizando acciones que propongan la gestión del riesgo como una solución posible para los desastres naturales y sociales.

Las instituciones /organismos involucrados pueden diferenciarse por grupos bien sectorizados, a saber:

\section{Escala Local:}

- Gobierno Local: Representado por la Comisión Municipal de "Volcán". Este es un organismo público, de 3ra. Categoría por la cantidad de habitantes de la localidad: 1605 según el Censo Nacional del INDEC (2010). En esta comisión, no se pudo acceder a información sobre la existencia de alguna Dirección/Secretaría que atienda cuestiones referidas a los riesgos ambientales, desastres, etc. Tampoco se identificaron planes municipales que tiendan a la planificación o el ordenamiento territorial.

\section{Escala Provincial:}

- Gobierno provincial, a través del Ministerio de Infraestructura, Servicios Públicos, Tierra y Vivienda y del Ministerio de Ambiente, se presentan como los organismos a nivel provincial, encargados de las políticas públicas que deberán seguir las municipalidades, comisiones municipales y la población en relación a las acciones de planificación y atención de los desastres:

- Ministerio de Infraestructura, Servicios Públicos, Tierra y Vivienda: Es el organismo encargado de realizar la planificación de la localidad. Ante el alud del año 2017, realizó acciones para una correcta planificación urbana, aunque ésta solo se limitó a la reconstrucción de viviendas para los damnificados. Este plan de refacción / reconstrucción de viviendas, fue realizado particularmente por el Instituto de 
Vivienda y Urbanismo de Jujuy (IVUJ), dependiente del ministerio mencionado con anterioridad. En cuestiones de planificación urbana y ordenamiento territorial (defensas, alejamiento, etc.) no se observan modificaciones relevantes.

- Ministerio de Ambiente: A través de la Dirección Provincial de Ordenamiento Ambiental Territorial, brindó asesoramiento en lo referido a cuestiones del uso ambientalmente adecuado de los recursos ambientales en el proceso de reconstrucción de las viviendas y del pueblo.

\section{Escala Nacional:}

- Gobierno Nacional: a través de los diferentes organismos nacionales el Estado Nacional busca atender a las problemáticas de la provincia en materia de riesgos y desastres. En este sentido, y con la aplicación del Sistema Nacional para la Gestión Integral del Riesgo (SINAGIR) que fue creado en octubre de 2016 tras la sanción de la Ley 27.287 el Gobierno Nacional, buscando minimizar los riesgos y trabajar para disminuir los desastres, propuso una serie de acciones a ser realizadas por las diferentes regiones, teniendo en cuenta los riesgos a los que se expone cada lugar. En este sentido, la provincia de Jujuy, y especialmente los riesgos de localidad de Volcán, están considerados dentro de las "Amenazas Geodinámicas" entendiendo que éstas son aquellas "que afectan seriamente al territorio y la población argentina. Son de tres tipos: remoción en masa, volcanes y terremotos" (SINAGIR, PNRRD. p. 26).

Ante esto, el Sistema explica que, debido a las características del ambiente y relieve, la localidad de Volcán se encontraría dentro de los:

Movimientos de masa de intensidad alta: Las personas están en peligro tanto en el exterior como en el interior de las viviendas o edificios. Existe un alto peligro de destrucción repentina de viviendas y edificios. (SINAGIR, PNRRD. p. 29)

Es por esto, que algunos de los organismos a nivel nacional que el Sistema plantea que deben ayudar y proponer estrategias son:

- Ministerio de Seguridad

- $\quad$ Servicio Geológico Minero Argentino

- Servicio Meteorológico Nacional

- $\quad$ Comisión Nacional de Actividades Espaciales

- Instituto Geográfico Nacional

En relación a la ley N²7.287 "Sistema Nacional para la Gestión Integral del Riesgo" es importante mencionar que en el caso de la provincia de Jujuy, existe una adhesión a la normativa nacional, mediante la Ley 6.035 publicada en el Boletín Oficial el 24 de noviembre de 2017. Esta adhesión propone y designa al Ministerio de Seguridad de la provincia de Jujuy como organismo de aplicación de la ley, evidenciando así una correspondencia con la ley nacional y las acciones propuestas.

\section{La comunicación del riesgo}


La comunicación de riesgos puede definirse como el intercambio de información entre partes interesadas acerca de las características de un evento, magnitud, recurrencia, dimensiones, significado o control del riesgo. Las partes interesadas son los agentes sociales involucrados pudiendo ser agencias gubernamentales, corporaciones, grupos industriales, sindicatos, medios de comunicación, medios científicos, organizaciones profesionales, grupos especiales y ciudadanos en particular.

De acuerdo con el momento del riesgo, es decir, el antes, durante y después, los canales de información pueden ser desde audiencias, reuniones, documentos, gacetillas y conferencias de prensa u otros. Respecto de la información sobre un desastre natural esta puede ser controlada por diversas fuentes tanto por observación directa y corroborada por otras observaciones directas

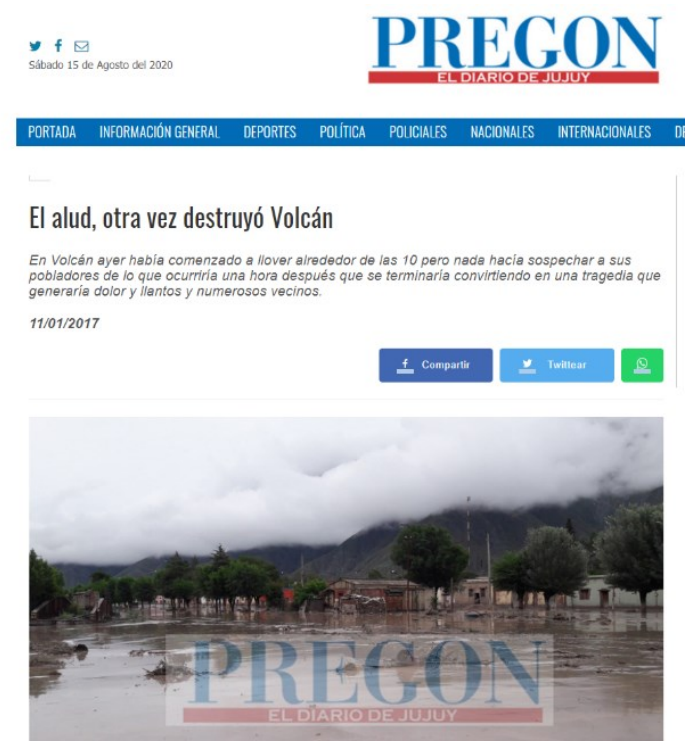

Figura N 10: Comunicación del evento en medios de comunicación - Fuente: Diario Pregón, Jujuy (2017)

Puede entenderse que, en el último alud de Volcán, la comunicación fue masiva: diversos medios provinciales e incluso nacionales presentaron el evento y la problemática que se desarrollaba. Además, posteriormente, las comunicaciones siguieron existiendo no solo para recordar la situación trágica, sino como una manera de sensibilizar a las poblaciones sobre los riesgos a los que están expuestos los pobladores de la Quebrada.

Es importante resaltar en este punto, la necesidad de visibilizar estas situaciones, tanto a nivel local como nacional, a fin de que se puedan establecer operaciones, tareas y propuestas válidas que permitan a la población conocer los riesgos, las amenazas y también las diferentes acciones y gestiones que se pueden realizar ante estas situaciones.

\section{Conclusiones y reflexiones finales}


Entender los conceptos referidos a los riesgos, amenazas y vulnerabilidad ambiental son muy importantes debido a que brindan a los profesionales del ambiente, un abanico de conceptualizaciones y herramientas que permiten analizar la realidad desde diversos puntos.

Conocer las tipologías de las amenazas, así como las condiciones de vulnerabilidad y exposición de la población, además de poder diferenciar éstas de los riesgos o de los desastres resulta muy provechoso. Por otro lado, es necesario entender que la bibliografía propuesta es pertinente debido a que analiza, desde diversos puntos y visiones, las realidades del ambiente.

Es importante decir que resulta imprescindible para los futuros profesionales formados en la dimensión del ambiente y del desarrollo sustentable analizar y atender a las situaciones donde se pone en juego el ambiente. A su vez, esta situación plantea un desafío para seguir trabajando en el conocimiento de los riesgos y la vulnerabilidad, entendiendo que los mismos forman parte del desarrollo ambiental y natural.

Además, es pertinente promover que estos conceptos se los debe relacionar con la realidad local en tanto en todos los ámbitos y contextos tenemos amenazas, riesgos e incluso vulnerabilidades y lamentablemente, frecuentemente estamos atravesados por fenómenos o desastres.

Por último, puede decirse que los fenómenos que suceden periódicamente en Volcán y en Quebrada de Humahuaca se presentan como consecuencia de eventos adversos, pero que las sociedades y los organismos especializados deben tener en cuenta los diferentes estudios y previsiones a fin de mitigar y minimizar los efectos negativos que esta problemática genera. No se puede pensar que se evitaran estos fenómenos pero si se puede sensibilizar a la población sobre los riesgos, educar a las diferentes generaciones acerca de cómo reaccionar ante estos desastres y de esa manera se podrá enfrentar estas situaciones de una manera adecuada.

\section{Referencias bibliográficas}

Albeck, M. E. y González, A. M. (1994). Quebrada de Humahuaca, más de 10.000 años de historia. Buenos Aires: Ministerio de Educación de la Nación.

Alonso, R. N. (2011). Riesgos geológicos en el norte argentino: terremotos, volcanes, avalanchas, inundaciones, desertización y otros fenómenos naturales. Salta, Argentina: Mundo Editorial.

Alonso, R.N. (2015). Geografía Física del Norte Argentino (Salta y Jujuy). Salta, Argentina: Mundo Editorial.

Alonso, R. N. (2019). Historia Natural y Cultural. Salta, Argentina: Mundo Editorial.

Blaikie, P. (1996). Vulnerabilidad, el entorno social, político y económico de los desastres. Bogotá, Colombia: La Red. 
Carballo, C. y Pereyra, A. (2012). Riesgos ambientales y eventos extremos, sequias en Buenos Aires. Segundas Jornadas Nacionales de Investigación y docencia en Geografía Argentina. Facultad de Ciencias Humanas, UNICEN, Tandil.

Castro, H. (2015). La trama reciente del riesgo ambiental en la Quebrada de Humahuaca. En: Riesgos al sur. Diversidad de riesgos de desastres en Argentina. Buenos Aires: Imago Mundi.

Cremonte, M. y Scaro, A. (2010). Consumo de vasijas cerámicas en un contexto publico tardío del Pucará de Volcán. En Revista do Museu de Arqueologia e Etnologia. São Paulo. Estrategia Internacional para la Reducción de Desastres (2004). Vivir con el Riesgo. Washington: ONU - EIRD.

Kirbus, F. B. (2006). Quebrada de Humahuaca. Colección Patrimonio de la Humanidad. Buenos Aires: Ediciones del Eclipse.

Lavell, A. y Franco, E. (1996). Degradación ambiental, riesgo y desastre urbano. Problemas y conceptos. Hacia la definición de una agenda de investigación. En: Ciudades en riesgo, degradación ambiental, riesgos urbanos y desastres. Lima: La Red.

Lavell, A. y Franco, E. (1996). Estado, sociedad y gestión de los Desastres en América Latina: en busca del paradigma perdido. Lima: La Red.

Montenegro, M. (2011). Itinerarios culturales y patrimonio religioso en la Quebrada de Humahuaca, Jujuy, Argentina. En: Ledesma R. y Castro V. F. (Ed). Caminos que unen historia, paisaje y turismo. Experiencias patrimoniales en Argentina y España. Badajoz, España: Editorial PSIQUE-EX.

Pereyra, A. B. (2009). Vulnerabilidad y riesgo ambiental. Buenos Aires: Universidad Nacional de Quilmes.

Pereyra, A. B. (2020). Vulnerabilidad y riesgo ambiental. Buenos Aires: Universidad Nacional de Quilmes.

Quintana, E. (2017). Vulnerabilidad hidrogeológica en la Quebrada de Humahuaca. En Revista Proyección 31. Jujuy: Colegio de Ingenieros de Jujuy.

Rodríguez Fernández, L. R.; Heredia, N; Seggiaro, R. E. y González M. (1999). Estructura andina de la Cordillera Oriental en el área de la Quebrada de Humahuaca, Provincia de Jujuy, NO de Argentina. Trabajos de Geología 21, pp. 321-332.

Romero, G. y Markrey, A. (1993). ¿Cómo entender los desastres naturales? En: Los desastres no son naturales. Colombia: La Red.

Savi, S., Schildgen, T., Tofelde, S., Wittmann, H., Scherler, D., Mey, J., Alonso, R. N., Strecker, M. R. (2016). Climatic controls on debris-flow activity and sediment aggradation: The Del Medio fan, NW Argentina. En Journal of Geophysical Research.

Tarbuck, E. J. y Lutgens, F. K. (2005). Ciencias de la Tierra. Una introducción a la geología física. Madrid: Pearson Educación. 
Villarrubia Gómez, A.P. (2012). Diseño y elaboración de un producto turístico religioso para la festividad de la virgen de la Candelaria, de Humahuaca, provincia de Jujuy, Argentina. (Tesis inédita). Universidad Católica de Santiago del Estero, sede San Salvador de Jujuy. Weigert, R. S. G.; Chalabe, S. A. y Maigua, L. E. (2010). Efectos de la intervención humana en la dinámica erosiva del río Grande de Jujuy entre Volcán-"Peña Azul" (departamento Tumbaya, provincia de Jujuy). En VI Jornadas de Ciencia y Tecnología de las Facultades de Ingeniería del NOA Publicación: Investigaciones en Facultades de Ingeniería del NOA-2010; (pp. 243-247).

Wilches Chaux, G. (1993). La vulnerabilidad global. En Maskrey, A. Los desastres no son naturales. Bogotá, Colombia: La Red.

Zilio M. C. (2019). Vivir al pie de los volcanes o el incierto encanto de habitar zonas peligrosas. Una aproximación desde la teoría social del riesgo. En VII Congreso Nacional de Geografía de Universidades Públicas y XXI Jornadas de Geografía de la UNLP. Universidad Nacional de La Plata. La Plata.

\section{Leyes/Reglamentaciones/Manuales}

CEPAL (2014) Manual para la Evaluación de Desastres. ONU: Chile.

Decreto 3021-G.2017. Gobierno de la provincia de Jujuy.

Documento "Plan Nacional Para La Reducción Del Riesgo De Desastres" 2018 - 2023

Ley Nacional N²7287. Sistema Nacional para la Gestión Integral del Riesgo y la Protección Civil, Buenos Aires, 28 de septiembre de 2011.

Ley Provincial $N^{\circ}$ 6035. Adhesión provincial a la Ley nacional 27.287 de "Creación del Sistema Nacional para la Gestión Integral del Riesgo y la Protección Civil", San Salvador de Jujuy, 24 de noviembre de 2017. 